\title{
The transition region above sunspots
}

\author{
Hui Tian * (D), Tanmoy Samanta and Jingwen Zhang
}

\begin{abstract}
Over decades, sunspots and their fine structures have been studied in detail at the photospheric level with different ground-based telescopes, as the surface of the Sun primarily emits light in the visible wavelengths. For a very long period, the upper atmosphere above the sunspot regions, especially the transition region (TR) above sunspots where the plasma emits light in the far ultraviolet (FUV) and extreme ultraviolet (EUV), has been poorly understood. In the past decades after the development of space instrumentations, FUV and EUV observations have uncovered many secrets of the TR above sunspots. In this paper, we present a brief review of research results about the TR structures and dynamics obtained through imaging and spectroscopic observations of sunspots in the past $\sim 20$ years. Though these observations have gathered remarkable and detailed information and greatly improved our understanding of the TR above the sunspots, paradoxically, they leave us with many new questions which should be answered in the future.
\end{abstract}

Keywords: Sunspots, Solar transition region, Chromosphere

\section{Introduction}

Sunspots are the darkest regions with the strongest magnetic fields and lowest temperatures on the surface of the Sun. Though observed by humanity for thousands of years, detailed investigation of the long-term evolution of sunspots started only 400 years ago, when Galileo Galilei invented the astronomical telescope. The number of sunspots strongly varies on a timescale of about 11 years, which is known as the sunspot cycle or solar cycle. Correspondingly, the occurring frequency of large-scale solar activities such as solar flares and coronal mass ejections also changes with such an 11-year cycle. As a result, the Earth's magnetosphere and ionosphere are disturbed by the same cycle. The total electromagnetic radiation of the Sun is also closely related to the 11-year sunspot cycle. For instance, the extreme ultraviolet (EUV) and far ultraviolet (FUV) emission of the Sun varies significantly over a solar cycle.

Modern high-resolution observations by large-aperture telescopes have revealed detailed substructures and short-term dynamics of sunspots. A sunspot often consists of a dark core termed umbra and a less dark

\footnotetext{
*Correspondence: huitian@pku.edu.cn

School of Earth and Space Sciences, Peking University, Beijing 100871, China
}

penumbra surrounding the umbra. Numerous umbral dots are often found inside the dark core, while the most prominent structures in the penumbra are the so-called penumbral filaments. Systematic outward flows, termed Evershed flows (Evershed 1909), are usually present in the penumbra. Some sunspots also have light bridges, which are bright lane-like structures dividing the umbra into two or more parts. It is believed that umbral dots, penumbral filaments, and light bridges are formed as a result of vigorous convective motions. For a detailed description of these substructures and dynamics, we refer to the comprehensive review by Solanki (2003). Some of these substructures and dynamics have been well reproduced through radiative magnetohydrodynamic (MHD) simulations (see a review by Rempel and Schlichenmaier 2011).

Most studies of sunspots are focused on the photospheric structures and dynamics. For a very long period, the upper atmosphere in sunspot regions, especially the transition region (TR) above the sunspots, has been poorly understood. This is mainly due to the fact that there were only very limited numbers of TR observations in the sunspot regions. By definition, the TR is the interface region between the chromosphere and corona, where the temperature increases from roughly $2 \times 10^{4}$ to $8 \times 10^{5} \mathrm{~K}$ (e.g., Tian 2017). TR probing relies mainly on 
imaging and spectroscopic observations of the EUV and FUV emission, primarily in the spectral range of 400$1600 \AA$. In the past $\sim 20$ years, observations from mainly three EUV/FUV spectrographs have greatly improved our understanding of the TR. The first two instruments are the Solar Ultraviolet Measurements of Emitted Radiation (SUMER, Wilhelm et al. 1995) and Coronal Diagnostic Spectrometer (CDS, Harrison et al. 1995) onboard the Solar and Heliospheric Observatory (SOHO) launched in late 1995. The SUMER instrument has a very broad wavelength coverage, from roughly 660 to $1610 \AA$ A. Hundreds of strong TR lines have been observed by SUMER. The spatial resolution of SUMER observations is about $2^{\prime \prime}-3^{\prime \prime}$. Unfortunately, most SUMER observations were performed in the first few years after the launch of $\mathrm{SOHO}$, when the solar activity was relatively low. As a result, there are limited numbers of sunspot observations by SUMER. For more than one solar cycle, spectra of some strong TR lines have been acquired in many CDS observations. However, the CDS instrument was mainly designed for coronal studies and the spatial resolution of the data is of much poorer quality than that of SUMER. Despite that, SUMER and CDS observations have revealed significant new insight into the structures and dynamics of the TR above sunspots. Our knowledge of the TR above sunspots has been greatly enhanced after the launch of the Interface Region Imaging Spectrograph (IRIS; De Pontieu et al. 2014) mission in June 2013. As a UV spectrograph with slit-jaw imaging (SJI) capability, IRIS has been performing both imaging and spectroscopic observations of the TR with unprecedented high resolution $\left(0.3^{\prime \prime}-0.4^{\prime \prime}\right)$ since July 2013 . The imaging capability of IRIS allows us to capture highly dynamic events occurring in the TR and chromosphere above the sunspots. The high spectral resolution permits the accurate detection of plasma flows with different speeds in the sunspots' atmosphere.

Here, we review our current knowledge of the TR structures and dynamics above sunspots, mainly based on observations from the SUMER, CDS and IRIS instruments. We will also discuss what new questions should be answered in the future to better our understanding of the TR above sunspots.

\section{Average properties of the TR above sunspots}

Figure 1 shows images of different layers of a sunspot's atmosphere. Emission structures of the photosphere and chromosphere appear to be similar, both revealing a dark umbra and a less dark penumbra. The IRIS $1400 \AA$ filter samples mainly the Si IV 1394 and $1403 \AA$ lines that are formed at a temperature of $8 \times 10^{4} \mathrm{~K}$ under the condition of ionization equilibrium. From the $1400 \AA$ image, we can see some patches of enhanced emission within

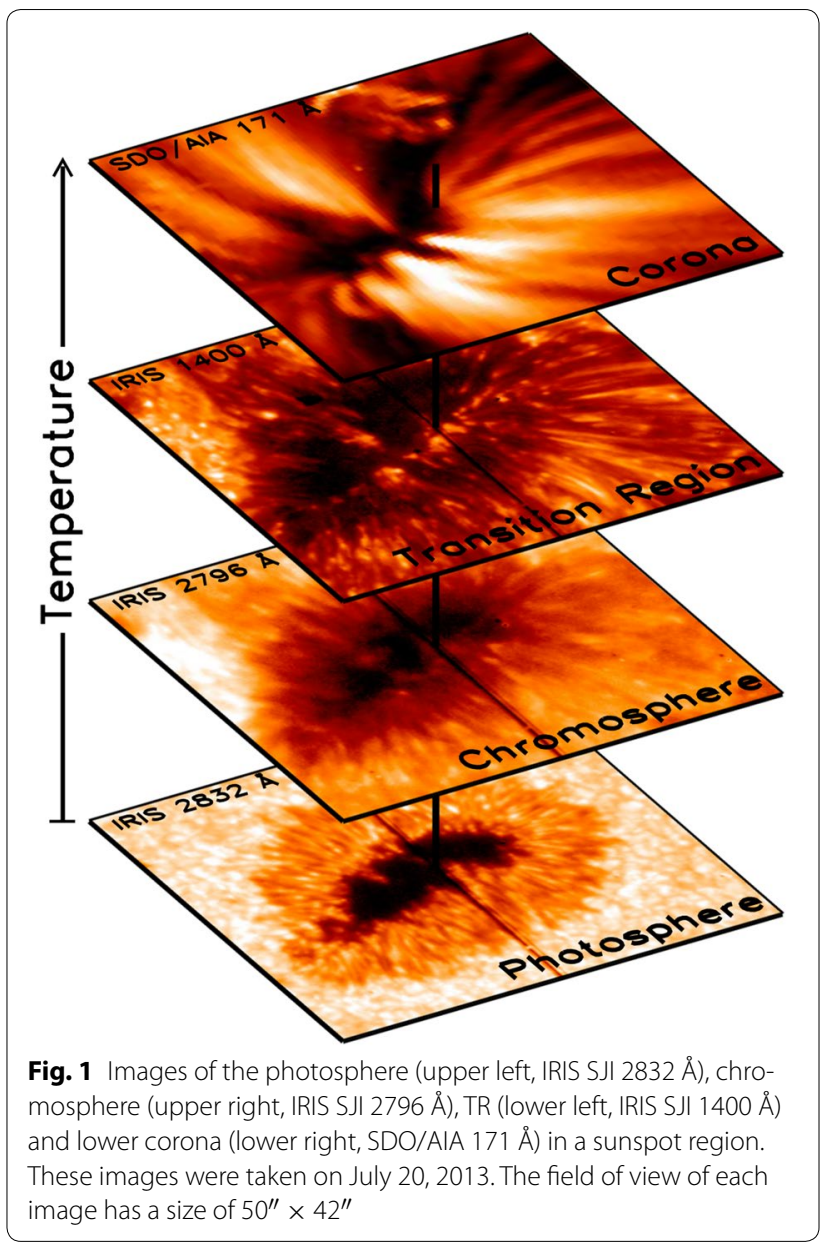

the umbra. Such enhanced TR emission features are very common in sunspot umbrae. The $171 \AA$ filter of the Atmospheric Imaging Assembly (AIA, Lemen et al. 2012) onboard the Solar Dynamics Observatory (SDO) samples emission from mainly several Fe X/Fe IX lines, which are formed around $9 \times 10^{5} \mathrm{~K}$ (lower corona). The $171 \AA$ image presented in Fig. 1 reveals some far reaching loops that appear to be rooted in the sunspot umbra.

Sunspot plumes are the most prominent features in the TR above sunspots. They often exhibit as compact and very bright features in the TR spectral lines within sunspots (Foukal et al. 1974; Foukal 1976; Solanki 2003). SUMER observations have revealed the presence of these plumes in a wide range of temperatures. Normally, these sunspot plumes are characterized by significantly enhanced intensities of spectral lines with formation temperatures between $10^{5}$ and $10^{6} \mathrm{~K}$ (in the upper TR and lower corona). Dammasch et al. (2008) suggested that sunspot plumes are just the common footpoints of many far reaching loops. These plumes are often found to be associated with significant redshifts of spectral lines formed in the upper TR such as the Ne VIII $770 \AA$ line 
(e.g., Brynildsen et al. 2001a; Marsch et al. 2004; Brosius and Landi 2005; Dammasch et al. 2008). The line profiles are often entirely redshifted by $\sim 30 \mathrm{~km} / \mathrm{s}$ at locations of sunspot plumes (see Fig. 2). Marsch et al. (2008) found that these redshifts are associated with the lower part of the legs of large-scale magnetic loops that are reconstructed based on magnetic field measurement in the photosphere. There are two proposed mechanisms for the generation of these downflows: by siphon flows along far reaching loops (Brynildsen et al. 2001a, b; Doyle and Madjarska 2003; Brosius 2005) or by cooling and condensing the coronal plasma (Noyes et al. 1985; Dammasch et al. 2008). However, direct evidence has not been observed for either of these mechanisms.

SUMER observations have revealed significant blueshifts in the umbrae of some sunspots. For instance,
Fig. 2 shows a patch of blueshift inside the umbra in both the C IV $1548 \AA$ and Ne VIII $770 \AA$ A lines that are formed at a temperature of about $10^{5}$ and $6 \times 10^{5} \mathrm{~K}$, respectively. The magnitude of the blueshift appears to increase with the formation temperature. Magnetic field extrapolation also indicates that the blueshifted region is associated with open field lines, suggesting that these blueshifts are possible signatures of the initial acceleration of the solar wind (Marsch et al. 2004).

Detailed plasma diagnostics of the TR above sunspots has been performed using the spectra acquired by EUV spectrographs such as SUMER and CDS. Tian et al. (2009) has carried out a comprehensive investigation of the electron densities at different locations of an active region using ten density-sensitive line pairs observed with SUMER. At a temperature of $\sim 10^{5.3} \mathrm{~K}$, they found

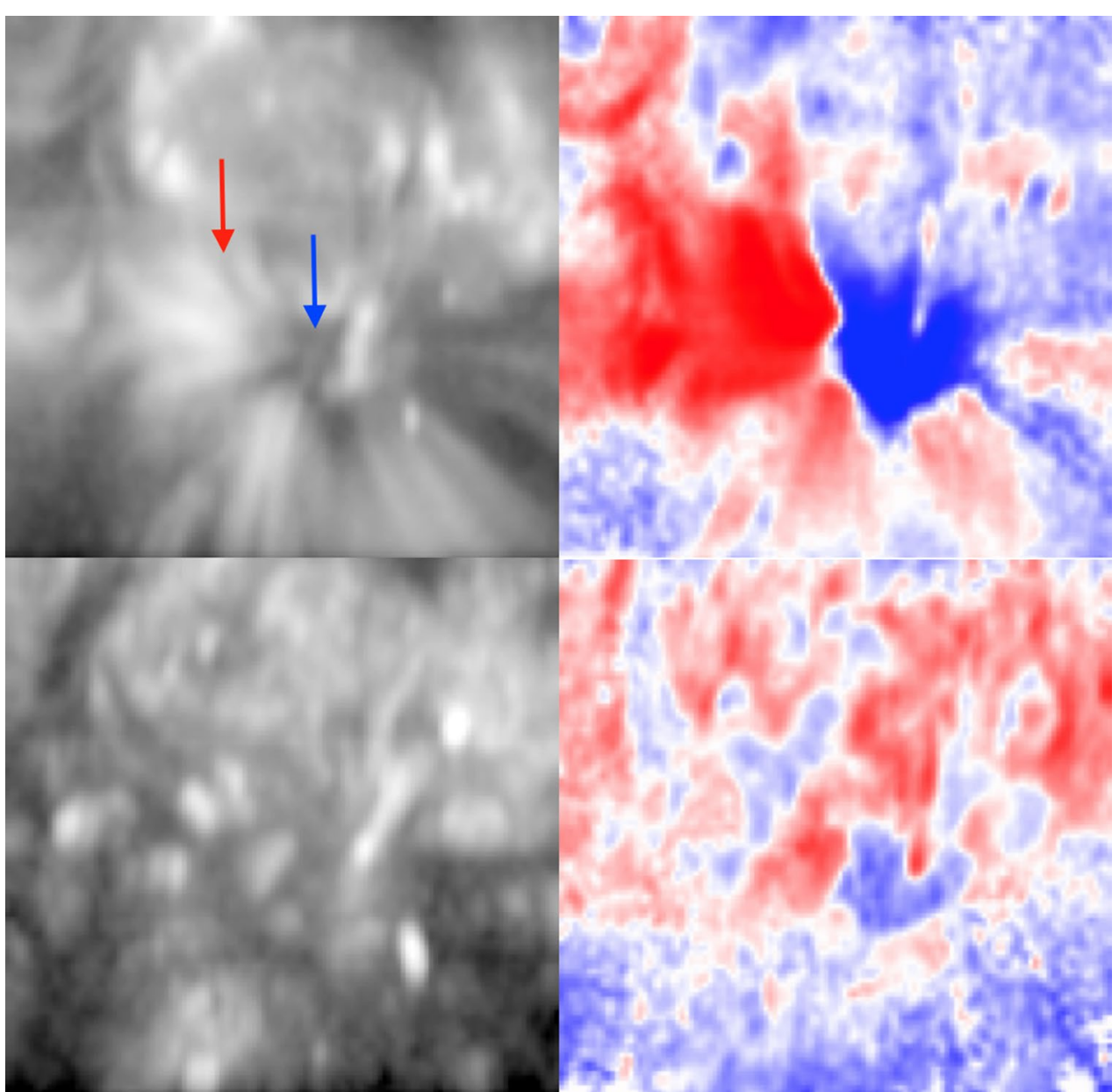

Fig. 2 Intensity images (left) and Dopplergrams (right) of two TR lines taken by SUMER on August 2, 1996. The upper and lower panels show the results for the Ne VIII $770 \AA$ and C IV $1548 \AA$ lines, respectively. The red and blue arrows indicate a sunspot plume and the umbra, respectively. The field of view of each image has a size of about $120^{\prime \prime} \times 120^{\prime \prime}$. These images are taken from SUMER Image Database: http://www2.mps.mpg.de/ projects/soho/sumer/text/s024503.html. The same dataset has also been analyzed by Marsch et al. (2004) 
a density of $\log \left(N_{\mathrm{e}} / \mathrm{cm}^{-3}\right)=10$ in the umbral regions and sunspot plumes, which is one order of magnitude smaller than in the plage and penumbra regions. Similar electron densities have also been derived in other sunspot plumes (Doyle et al. 1985; Doyle and Madjarska 2003). The electron densities of umbrae and sunspot plumes appear to be similar to the typical density of normal quiet Sun regions at similar temperatures (Brosius and Landi 2005).

Using multiple emission lines formed at a wide range of temperatures, the differential emission measure (DEM) of the sunspot atmosphere can be derived. Brosius and Landi (2005) found that the DEM curves of two sunspot plumes exhibit peaks at $\sim 10^{5.6}$ and $\sim 10^{5.8} \mathrm{~K}$, respectively. Tian et al. (2009) compared the DEM curves of different regions and found that the DEM curves of the umbra, penumbra and plage regions all peak at a similar temperature around $10^{5.75} \mathrm{~K}$. They also found that the DEM curve of the sunspot plume region peaks at a much lower temperature, $\sim 10^{5.45} \mathrm{~K}$. The temperature structure revealed through the DEM analysis is consistent with the fact that sunspot plumes usually show greatly enhanced emission of spectral lines formed in the upper TR. For a long time it was unknown why the plume emission was so prominent at upper TR temperatures. Through a detailed analysis of the SUMER spectra, Tian et al. (2009) concluded that the enhanced TR emission of sunspot plumes is most likely caused by a large filling factor.

Plasma diagnostics using the SUMER data also led Tian et al. (2009) to propose that the TR above sunspots is likely higher and more extended compared to the TR above the plage regions. Such a scenario is illustrated in Fig. 18 of Tian et al. (2010). This scenario is consistent with the temperature structure of sunspots proposed by Nicolas et al. (1982), in which the temperature in the TR above sunspots is lower than the surrounding temperature at the same height.

\section{Dynamics in the TR above sunspots}

Early observations of the TR above sunspots occasionally revealed fast downflows with speeds of $\sim 100 \mathrm{~km} / \mathrm{s}$ (e.g., Brekke 1993; Brynildsen et al. 2001b, 2004a). These downflows are often seen in spectral lines with a formation temperature around $10^{5.0} \mathrm{~K}$. The flow speeds obviously exceed the sound speed at such a temperature; thus, these flows are clearly supersonic. These supersonic downflows appear to be long-lasting rather than transient features. The spectral line profiles often exhibit two components, one is nearly stationary and the other is highly redshifted. Due to the relatively low spectral resolution in early observations, these two components are usually not well separated. The TR spectra acquired by IRIS have a much higher resolution. As result, these two components are often found to be well separated, just like two well-isolated emission lines. Such line profiles allow an accurate determination of the parameters of the two components through a double Gaussian fit. In addition, IRIS was launched around the solar maximum and has observed a very large number of sunspots, which allows comprehensive statistical investigations of these supersonic downflows. A recent statistical study by Samanta et al. (2018) found that these supersonic downflows are very common for sunspots. They also found that the electron density of the downflow component is obviously lower than that of the stationary component. Different from the primary component, no obvious oscillation has been found for the downflow component (Tian et al. 2014a; Straus et al. 2015). These results suggest that the supersonic downflows and the background TR are independent. Previous observations appear to show that these supersonic downflows are seen in emission lines formed only in the middle TR, in the temperature range of $10^{4.5}-10^{5.5} \mathrm{~K}$. However, Samanta et al. (2018) showed that some of these downflows can also be identified in the chromospheric Mg II $\mathrm{k}$ and $\mathrm{h}$ lines. The nature and formation mechanisms of these downflows remain unclear. Recent investigations by Straus et al. (2015) and Chitta et al. (2016) suggested that these persistent supersonic downflows may produce stationary shocks. It is worth noting that IRIS also observed a different type of supersonic downflows, which are bursty rather than steady (Kleint et al. 2014). These downflows can have speeds up to $\sim 200 \mathrm{~km} / \mathrm{s}$ in several emission lines from the ions of Mg II, C II and Si IV. These transient supersonic downflows have been suggested to be coronal rain falling into sunspots.

Sunspots are known to exhibit periodic oscillations in both the photosphere and chromosphere. The 3-min oscillations detected in the chromosphere of sunspots are often suggested to be leakage of $p$-mode waves from the photosphere. Using SUMER and CDS observations of sunspots, 3-min oscillations have also been identified in TR lines, suggesting the propagation of the waves further up to the TR (Brynildsen et al. 1999a, b, 2004b; Maltby et al. 1999; O'Shea et al. 2002). Some of these studies found signatures of nonlinearity for these waves. Thanks to the high resolution of IRIS observations, waves are clearly observed in both the 1330 and $1400 \AA$ slit-jaw images of IRIS. These passbands sample mainly the TR emission from two C II and Si IV lines in sunspots. Tian et al. (2014a) performed detailed analysis of the TR spectra and identified strong signals of shock waves in the TR above sunspots. IRIS observations of these shocks at different layers appear to be consistent with the scenario of upward propagating magnetoacoustic shock waves along inclined magnetic field lines (Madsen et al. 2015). 
A recent study by Zhao et al. (2016) suggests that these waves can propagate further up into the corona.

Another type of oscillatory phenomenon in sunspots is the surge-like up-and-down motion above some light bridges (Asai et al. 2001; Shimizu et al. 2009; Louis et al. 2014; Toriumi et al. 2015). These surges are usually observed in chromospheric images. Due to the lack of imaging of the TR above sunspots, for a long time it was unclear whether these chromospheric surges affect the overlying TR or not. Based on IRIS observations, Rezaei (2018) concluded that light bridges consist of multithermal structures extending up to the TR. Thanks to the direct imaging of the TR by IRIS, we now can study the TR response of these surges in detail. In 2015, Yang et al. (2015) and Bharti (2015) independently identified a bright front with TR temperatures ahead of the chromospheric surges above a light bridge. The bright front can be seen in the 1330 and $1400 \AA$ slit-jaw images of IRIS, while the wall-like surges are visible in chromospheric

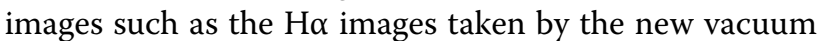
solar telescope (NVST, Liu et al. 2014) and the $2796 \AA$ slit-jaw images taken by IRIS. Figure 3 shows an example of the surge and the bright front ahead of the surge. Zhang et al. (2017) analyzed the kinematics of such a bright front in another light bridge observation by IRIS, and found parabolic trajectories of the bright front. They also found a linear correlation between the maximum velocity and deceleration of the motion of the bright front, suggesting the nature of these long-lasting oscillatory motions to be slow-mode shock waves. The bright front may be caused by heating to TR temperatures through either a shock front or compression when the chromospheric surges hit the overlying atmosphere. It is worth mentioning that previously magnetic reconnection was usually suggested to trigger these surges (Asai et al. 2001; Shimizu et al. 2009; Louis et al. 2014; Toriumi et al. 2015). IRIS observations have also revealed occasionally occurring reconnection events at light bridges (e.g., Tian 2018; Song et al. 2017; Hou et al. 2017). Reconnection can heat the local plasma to typical TR temperatures at light bridges.

Direct imaging of the TR through the 1330 and $1400 \AA$ filters of IRIS has revealed many sub-arcsec bright dots in sunspots, mostly in the penumbra (Tian et al. 2014b). Figure 4 shows an $1400 \AA$ image of a sunspot, where some small-scale bright dots are clearly present. These bright dots have a lifetime mostly less than $1 \mathrm{~min}$, and they often show clear signatures of enhanced Si IV emission. The Si IV line profiles are also obviously broadened. Many of these bright dots appear to be located at the footpoints of coronal loops. Tian et al. (2014b) also estimated the thermal energy of a typical bright dot, which turned out to be of the same order of nanoflares. They suggested that some of these bright dots are associated with small-scale energy release events at the TR footpoints of magnetic loops. From the $193 \AA$ images taken

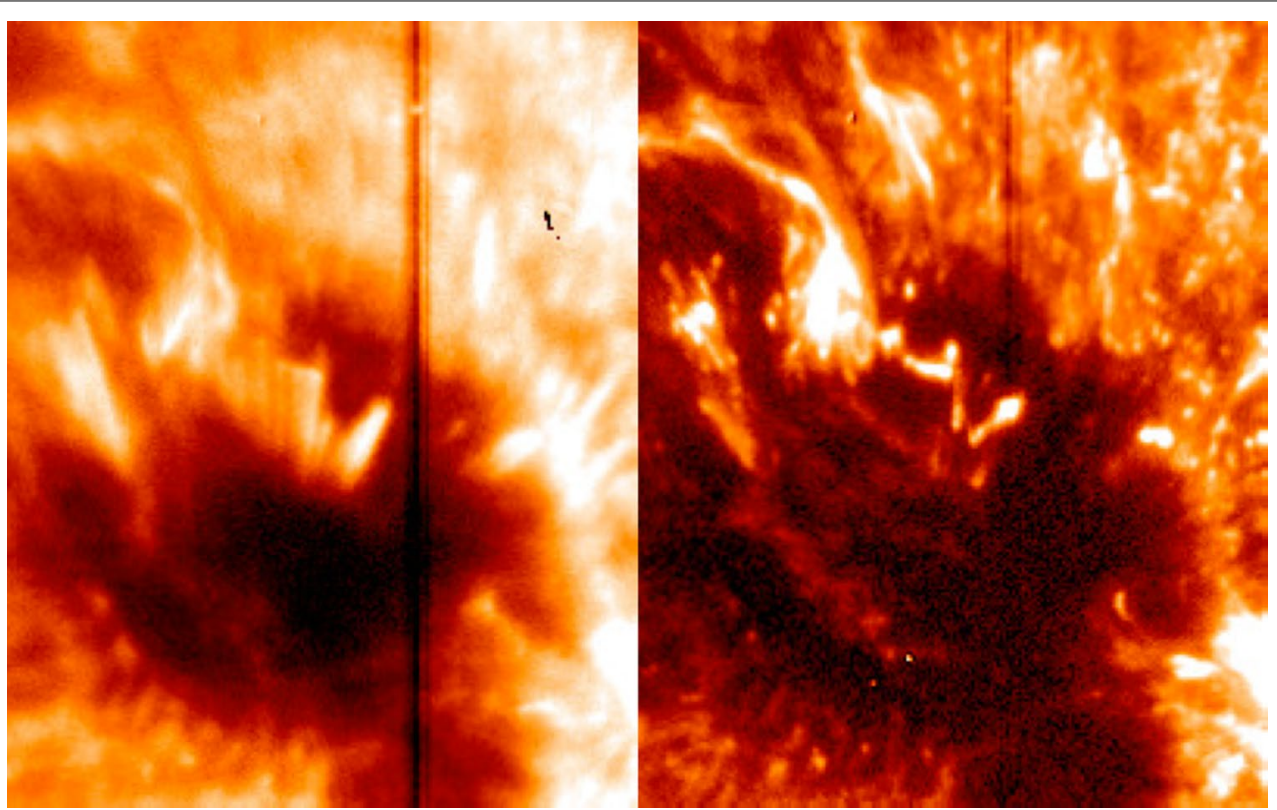

Fig. 3 Images of the chromosphere (left, IRIS SJI $2796 \AA$ ) and TR (right, IRIS SJI $1400 \AA$ ) in a sunspot region. These images were taken on July 20 , 2013. The field of view of each image has a size of $34^{\prime \prime} \times 42^{\prime \prime}$. Image reproduced with permission from Zhang et al. (2017), copyright by AAS 


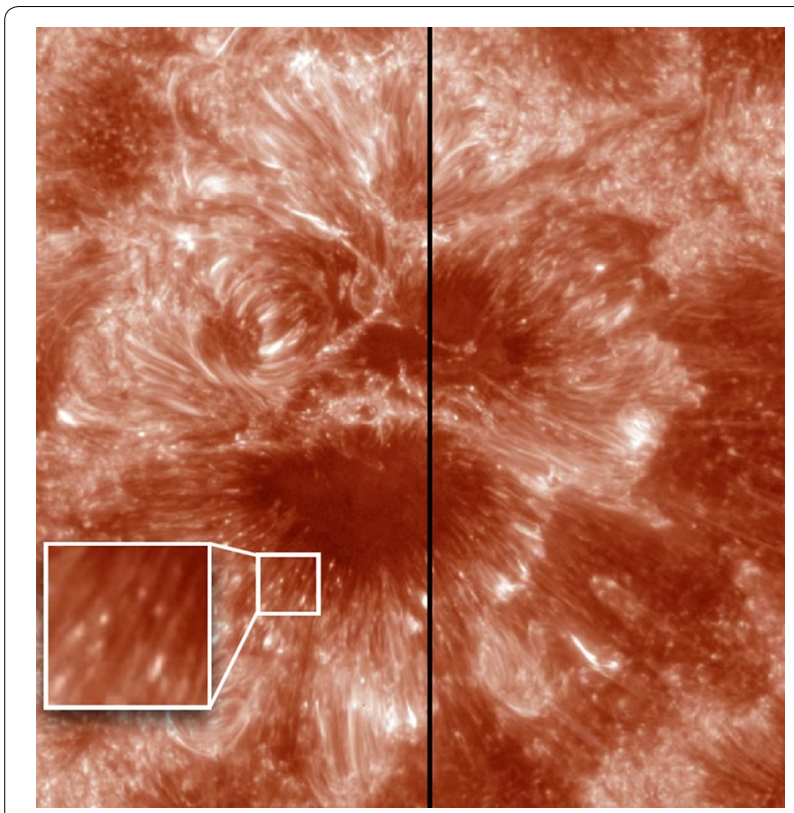

Fig. 4 A $1400 \AA$ image of a sunspot taken by IRIS on Nov 19, 2014 The field of view of the image has a size of about $160^{\prime \prime} \times 165^{\prime \prime}$. The small white box indicates a region where several penumbral bright dots (Tian et al. 2014b) are observed. The large white box shows a zoomed-in view of this region

by the High Resolution Coronal Imager (Hi-C) rocket flight, Alpert et al. (2016) also identified similar bright dots and attributed the visibility of these TR bright dots in the $193 \AA$ imaged to the TR contribution to the filter. These observations appear to suggest that these bright dots do not have coronal counterparts. Simultaneous observations in the chromosphere and TR (Vissers et al. 2015; Samanta et al. 2017) show that some of these TR bright dots rest at the tips of chromospheric penumbral micro-jets (Katsukawa et al. 2007; Tiwari et al. 2016). Vissers et al. (2015) suggested that these TR bright dots are the heating signature of the penumbral micro-jets, whereas Samanta et al. (2017) showed that the majority of the TR dots originate before the generation of the chromospheric jets. These observations left us with an unclear and confused understanding about their origination process and indicated the further need for high-quality imaging and spectroscopic observations. Interestingly, many bright dots show no detectable signatures in chromospheric images, which might be caused by cooling downflows from the overlying corona (Deng et al. 2016, Samanta et al. 2017). Another category of small-scale brightenings observed with IRIS is narrow-line-width UV bursts (Hou et al. 2016). The Si IV line profiles at locations of these bursts are very narrow, though their intensity is greatly enhanced. The formation mechanism of these bursts is still unclear.

\section{Future perspectives}

We have presented a brief review of our current understanding of the TR above sunspots. Undoubtedly, highresolution EUV and FUV observations in the past decades have greatly enriched our knowledge about the TR, especially the lower TR, above sunspots. In the meantime, these observations also pose new key questions which should be answered in the future. Some of these questions are listed below:

1. What is the formation mechanism of the $\sim 30 \mathrm{~km} / \mathrm{s}$ redshifts at footpoints of sunspot plumes?

2. What is the formation mechanism of the persistent supersonic downflows? How do these downflows contribute to the mass and energy budget of sunspots?

3. Can we use observations of sunspot oscillations at different heights to diagnose the physical parameters in the sunspot's atmosphere?

4. Why are the surge-like oscillations seen above some light bridges but not others?

5. How do the sub-arcsecond TR bright dots originate, and are they important for the heating of the upper atmosphere above sunspots?

6. How do the TR fine structures and fast dynamics affect the chromosphere and corona above sunspots?

Finally, it is worth noting that our knowledge about the upper TR $\left(10^{5}-10^{5.9} \mathrm{~K}\right)$ is poor due to the lack of direct imaging and high-resolution spectroscopic observations in this temperature regime. To improve our understanding of the coupling between the corona and the lower atmosphere, probing the upper TR should be one focus of future solar missions. We refer to Tian (2017) for a detailed discussion about this.

\section{Authors' contributions}

HT wrote the first draft of the manuscript. TS and JZ commented on and revised the manuscript. TS also prepared Figs. 1, 2, 3, and 4. All authors read and approved the final manuscript.

\section{Acknowledgements}

The SUMER project was financially supported by DLR, CNES, NASA and the ESA PRODEX programme (Swiss contribution). IRIS is a NASA small explorer mission developed and operated by LMSAL with mission operations executed at NASA Ames Research Center and major contributions to downlink communications funded by ESA and the Norwegian Space Centre.

\section{Competing interests}

The authors declare that they have no competing interests.

\section{Availability of data and materials}

The SUMER data are archived at the Max Planck Institute for Solar System Research in Göttingen, Germany. The IRIS data are freely available through the website of http://iris.Imsal.com/search/, which is managed by the Lockheed Martin Solar and Astrophysics Laboratory (LMSAL). The publicly available SDO/ AIA data are archived at Joint Science Operations Center (http://jsoc.stanford. edu/AIA/AIA_jsoc.html). 


\section{Consent for publication}

Not applicable.

\section{Ethics approval and consent to participate}

Not applicable.

\section{Funding}

This work was supported by the Recruitment Program of Global Experts of China and the Max Planck Partner Group program.

\section{Publisher's Note}

Springer Nature remains neutral with regard to jurisdictional claims in published maps and institutional affiliations.

Received: 27 November 2017 Accepted: 10 January 2018

Published online: 05 February 2018

\section{References}

Alpert SE, Tiwari SK, Moore RL, Winebarger AR, Savage SL (2016) Hi-C observations of sunspot penumbral bright dots. Astrophys J 822:35

Asai A, Ishii TT, Kurokawa H (2001) Plasma ejections from a light bridge in a sunspot umbra. Astrophys J 555:L65

Bharti L (2015) Fine structure above a light bridge in the transition region and corona. Monthly Notic R Astron Soc 452:L16

Brekke P (1993) An ultraviolet spectral atlas of the sun between 1190-1730 A Astrophys J Suppl 87:443

Brosius JW (2005) Mass flows in a disappearing sunspot plume. Astrophys J 622:1216

Brosius JW, Landi E (2005) Properties of a sunspot plume observed with the coronal diagnostic spectrometer aboard the solar and heliospheric observatory. Astrophys J 632:1196

Brynildsen N, Kjeldseth-Moe O, Maltby P, Wilhelm K (1999a) Nonlinear sunspot transition region oscillations in NOAA 8378. Astrophys J Lett 517:L159

Brynildsen N, Leifsen T, Kjeldseth-Moe O, Maltby P (1999b) Sunspot transition region oscillations in NOAA 8156. Astrophys J Lett 511:L121

Brynildsen N, Maltby P, FredvikT et al (2001a) Sunspot plumes and flow channels. Sol Phys 198:89

Brynildsen N, Maltby P, Kjeldseth-Moe O, Wilhelm K (2001b) Dual flows and oscillations in the sunspot transition region. Astrophys J Lett 552:L77

Brynildsen N, Maltby P, Kjeldseth-Moe O, Wilhelm K (2004a) Dual flows with supersonic velocities in the sunspot transition region. Astrophys $J$ 612:1193

Brynildsen N, Maltby P, Foley CR, Fredvik T, Kjeldseth-Moe O (2004b) Oscillations in the umbral atmosphere. Sol Phys 221:237

Chitta LP, Peter H, Young PR (2016) A closer look at a coronal loop rooted in a sunspot umbra. Astron Astrophys 587:A20

Dammasch IE, Curdt W, Dwivedi BN et al (2008) The redshifted footpoints of coronal loops. Ann Geophys 26:2955

De Pontieu B, Title AM, Lemen JR et al (2014) The interface region imaging spectrograph (IRIS). Sol Phys 289:2733

Deng N, Yurchyshyn V, Tian H et al (2016) Multi-wavelength study of transition region penumbral subarcsecond bright dots using IRIS and NST. Astrophys J 829:103

Doyle JG, Madjarska MS (2003) Sunspot plume observations in the EUV. The gas pressure differential between the umbra and surrounding region. Astron Astrophys 407:L29

Doyle JG, Raymond JC, Noyes RW et al (1985) The extreme ultraviolet spectrum of sunspot plumes-part two-spectral diagnostics and implications for cooling. Astrophys J 297:816

Evershed J (1909) Radial movement in sun-spots. Monthly Notices R Astron Soc 69:454

Foukal PV (1976) The pressure and energy balance of the cool corona over sunspots. Astrophys J 210:575

Foukal PV, Huber MCE, Noyes RW et al (1974) Extreme-ultraviolet observations of sunspots with the Harvard spectrometer on the Apollo telescope mount. Astrophys J 193:L143
Harrison RA, Sawyer EC, Carter MK et al (1995) The coronal diagnostic spectrometer for the solar and heliospheric observatory. Sol Phys 162:233

Hou Z, Huang Z, Xia L et al (2016) Narrow-line-width UV bursts in the transition region above sunspots observed by IRIS. Astrophys J 829:L30

Hou Y, Zhang J, Li T et al (2017) Simultaneous observations of $p$-mode light walls and magnetic reconnection ejections above sunspot light bridges. Astrophys J Lett 848:L9

Katsukawa Y, Berger TE, Ichimoto K et al (2007) Small-scale jetlike features in penumbral chromospheres. Science 318:1594

Kleint L, Antolin P, Tian H et al (2014) Detection of supersonic downflows and associated heating events in the transition region above sunspots. Astrophys J 789:L42

Lemen JR, Title AM, Akin DJ et al (2012) The atmospheric imaging assembly (AIA) on the solar dynamics observatory (SDO). Sol Phys 275:17

Liu Z, Xu J, Gu B-Z et al (2014) New vacuum solar telescope and observations with high resolution. Res Astron Astrophys 14:705

Louis RE, Beck C, Ichimoto K (2014) Small-scale chromospheric jets above a sunspot light bridge. Astron Astrophys 567:A96

Madsen CA, Tian H, DeLuca EE (2015) Observations of umbral flashes and running sunspot waves with the interface region imaging spectrograph. Astrophys J 800:129

Maltby P, Brynildsen N, Fredvik T, Kjeldseth-Moe O, Wilhelm K (1999) On the sunspot transition region. Sol Phys 190:437

Marsch E, Wiegelmann T, Xia L-D (2004) Coronal plasma flows and magnetic fields in solar active regions, combined observations from $\mathrm{SOHO}$ and NSO/Kitt peak. Astron Astrophys 428:629

Marsch E, Tian H, Sun J, Curdt W, Wiegelmann T (2008) Plasma flows guided by strong magnetic fields in the solar corona. Astrophys J 685:1262

Nicolas KR, Kjeldseth-Moe O, Bartoe J-DF, Brueckner GE (1982) High resolution EUV structure of the chromosphere-corona transition region above a sunspot. Sol Phys. 81:253

Noyes RW, Raymond JC, Doyle JG et al (1985) The extreme ultraviolet spectrum of sunspot plumes. I_observations. Astrophys J 297:805

O'Shea E, Muglach K, Fleck B (2002) Oscillations above sunspots: evidence for propagating waves? Astron Astrophys 387:642

Rempel M, Schlichenmaier R (2011) Sunspot modeling: from simplified models to radiative MHD simulations. Living Rev Sol Phys 8:3

Rezaei R (2018) Structure of sunspot light bridges in the chromosphere and transition region. Astron Astrophys 609:A73

Samanta T, Tian H et al (2018) Statistical investigation of supersonic downflows in the transition region above sunspots. Astrophys J (submitted)

Samanta T, Tian H, Banerjee D, Schanche N (2017) Dynamics of subarcsecond bright dots in the transition region above sunspots and their relation to penumbral micro-jets. Astrophys J 835:L19

Shimizu T, Katsukawa Y, Kubo M et al (2009) Hinode observation of the magnetic fields in a sunspot light bridge accompanied by long-lasting chromospheric plasma ejections. Astrophys J 696:L66

Solanki SK (2003) Sunspots: an overview. Astron Astrophys Rev 11:153

Song D, Chae J, Kwak H et al (2017) Chromospheric plasma ejections in a light bridge of a sunspot. Astrophys J Lett 835:240

Straus T, Fleck B, Andretta V (2015) A steady-state supersonic downflow in the transition region above a sunspot umbra. Astron Astrophys 582:A116

Tian $\mathrm{H}$ (2017) Probing the solar transition region: current status and future perspectives. Res Astron Astrophys 17:110

Tian $\mathrm{H}$ et al (2018) Frequently occurring reconnection jets from sunspot light bridges. Astrophys J (in press)

Tian H, Curdt W, Teriaca L, Landi E, Marsch E (2009) Solar transition region above sunspots. Astron Astrophys 505:307

Tian H, Marsch E, Tu C, Curdt W, He J (2010) New views on the emission and structure of the solar transition region. New Astron Rev 54:13

Tian H, DeLuca E, Reeves KK et al (2014a) High-resolution observations of the shock wave behavior for sunspot oscillations with the interface region imaging spectrograph. Astrophys J 786:137

Tian H, Kleint L, Peter H et al (2014b) Observations of subarcsecond bright dots in the transition region above sunspots with the interface region imaging spectrograph. Astrophys J 790:L29

Tiwari SK et al (2016) Transition-region/coronal signatures and magnetic setting of sunspot penumbral jets: hinode (SOT/FG), Hi-C, and SDO/AIA observations. Astrophys J 816:92 
Toriumi S, Katsukawa Y, Cheung MCM (2015) Light bridge in a developing active region. I. Observation of light bridge and its dynamic activity phenomena. Astrophys J 811:137

Vissers GJM, Rouppe van der Voort LHM, Rutten RJ et al (2015) Ellerman bombs at high resolution. III. Simultaneous observations with IRIS and SST. Astrophys J 812:11

Wilhelm K, Curdt W, Marsch E et al (1995) SUMER—solar ultraviolet measurements of emitted radiation. Sol Phys 162:189
Yang S, Zhang J, Jiang F, Xiang Y (2015) Oscillating light wall above a sunspot light bridge. Astrophys J 804:L27

Zhang J, Tian H, He J, Wang L (2017) Surge-like oscillations above sunspot light bridges driven by magnetoacoustic shocks. Astrophys I 838:2

Zhao J et al (2016) Observational evidence of magnetic reconnection for brightenings and transition region arcades in IRIS observations. Astrophys J Lett 830:L17

\section{Submit your manuscript to a SpringerOpen ${ }^{\circ}$ journal and benefit from:}

- Convenient online submission

- Rigorous peer review

- Open access: articles freely available online

- High visibility within the field

- Retaining the copyright to your article

Submit your next manuscript at $\boldsymbol{\nabla}$ springeropen.com 